\title{
OBJECTIONABLE IMAGE DETECTION IN CLOUD COMPUTING PARADIGM-A REVIEW
}

\author{
${ }^{1,2,3}$ Rashed Mustafa and ${ }^{1,2,4,5}$ Dingju Zhu \\ ${ }^{1}$ Laboratory for Smart Computing and Information Science, \\ Shenzhen Institutes of Advanced Technology, Chinese Academy of Sciences, China \\ ${ }^{2}$ University of Chinese Academy of Sciences, Beijing, China \\ ${ }^{3}$ Department of Computer Science and Engineering, University of Chittagong, Bangladesh \\ ${ }^{4}$ Shenzhen Public Platform for Triple-play Video Transcoding Center, China \\ ${ }^{5}$ School of Computer Science, South China normal University Guangzhou, China
}

Received 2013-10-08, Revised 2013-11-13; Accepted 2013-11-18

\begin{abstract}
Obscenity detection from images and videos are now crucial due to social and ethical reasons. It has been two decades the research on this field started. Most of the works are based on skin color detection, which are not suitable for finding obscenity. The reason for this is that, there are many skins like objects such as beach photos, human skin like animal's fur, skin colored painting that enables false positive and negative rate. In addition all works performed well on some particular set of images or video data. In this research some aspects of obscenity detection is described delineating strength, weakness and possible extensions of prior works. Introducing some new features and incorporation of multiple classifiers and transfer learning will lead the work more robust. Moreover, traditional multimedia cloud computing has been investigated in this study and proposed some new research ideas.
\end{abstract}

Keywords: Content Based Pornography Detection (CBPD), Content Based Image Processing (CBIP), Transfer Learning (TL), Multimedia Cloud Computing (MCC)

\section{INTRODUCTION}

There is an enormous number of online resource monitoring cells all over the world due to rapid development of Information and Communication Technology (ICT). In spite of those security systems, it is not possible to check all content based image or video appropriately. This is a threat for the Internet users while using computers in office or in front of family members including children. Moreover objectionable content contradicts social and ethical issues. Hence content based image and video processing especially for identifying obscenity has now been a challenging research area Jaganathan and Vennila (2013) and Geetha and Narayanan (2008). It has been almost two decades when Fleek et al. (1996) published the first paper in this issue on "Finding Naked People". After that a large number of works have been accomplished by different researchers all over the world. Text based protection system has been used in early 2000 for screening malicious contents from the Internet Kjeldsen and Kender (1996) and Yogarajah et al. (2010). Objectionable contents but non-objectionable web site names could not identify with these techniques. In support of this drawback, we can find millions of spaces that contain objectionable materials. Malicious contents can be categorized here as objectionable images. Most of the works are based on region based skin color detection which has low recognition rate whether an image contain obscenity in general sense. In this study we will focus on four subject matters following a new paradigm of using cloud computing in Content Based Pornography Detection (CBPD) Hadoop, 2013 Almeer (2012); Rossotti (1983); Kakumanu et al. (2007); RamosPollan et al. (2012) and Pereira et al. (2010):

Corresponding Author: Rashed Mustafa, Laboratory for Smart Computing and Information Science, Shenzhen Institutes of Advanced Technology, Chinese Academy of Sciences 
- Skin detection

- Erotogenic body parts detection Usages of different machine learning tools (SVM and Adaboost)

- Usage of Cloud Computing for Obscenity detection

The remaining parts of this article can be organized according to the following ways: section 2 briefly describes literature review; different color space models related to skin detection will be elucidated in section 3 and 4 . In section 5 a comparative evaluation of existing works has been evaluated, in section 6 some open research issues are proposed and finally a comprehensive discussion is presented in section 7 .

\section{LITERATURE REVIEW}

There are extensive literatures on obscenity detection but in this paper some significant works have taken into consideration. Previous research mainly focused on large connected skin regions, erotogenic organs and feature descriptors applied with some different classifiers.

The following figure (Fig. 1) demonstrates number of significant works published since 1996 to till now. It has been observed that in 2010 most of the papers published due to significant improvement of machine learning tools.

\section{SKIN DETECTION}

Skin detection is a bit challenging task due to large variations in appearance, color and shape, occlusion, intensity, location of light source Kjeldsen and Kender (1996); Yogarajah et al. (2010); Yin et al. (2011); Abadpour and Ksaei (2005); Vezhnevets et al. (2003) and Kakumanu et al. (2007). Noise can appear as speckles of skin like color and many other objects for example wood, cooper and some clothes that are often confused as skin Wong et al. (2003) and Kakumanu et al. (2007). The noise can also be occurred by illumination that is the change of light source distribution and the illumination level (indoor, outdoor, highlights, shadows, non-white-lights) produces a change in the color. Illumination for the same person can be differed using different camera. Human skin color can be varied from person to person due to ethnicity. As for example skin color for the people of Asian differs with African, Caucasian and Hispanic groups. Some other factors such as age, sex, body parts, makeup, hairstyle, costumes, background colors, shadows, motion also affects the skin color appearance Kakumanu et al. (2007). In general, human skin is characterized by a combination of red and melanin (yellow and brown) and there is somewhat a range of hue for skin and saturation that represent skinlike pixels Taqa and Jalab (2010) and Rossotti (1983).

\subsection{Color Based Skin Detection}

When the skin is yellowish the saturation is more with much melanin Rossotti (1983). The main goal of skin detection and classification is to build a decision rule that discriminate between skin and non-skin pixels. Identifying skin color pixels involves finding a range of values for which most skin pixels would fall in a given color space Alshamasin et al. (2009); Brown et al. (2001) and Caetano and Barone (2001). The target is for a high detection rate and low false positive rate.

Fleek et al. (1996) demonstrates an automatic system for telling whether there are naked people present in the image. The strategy includes color and texture properties to obtain a mask for skin regions. It is effective for a wide range of shades and colors of skin. Skin regions are then fed to a specialized grouper, which attempts to group a human figure using geometric constraints on human structure. Using RGB color model and lack of machine learning tool are the main limitations.

Kjeldsen and Kender (1996) defined a color predicate in HSV color space for separating the hand from a cluttered background. The segmentation algorithm has also been applied to assist a user extract objects from the background in arbitrary color imagery. The method has shown its flexibility in a range of different office environments, segmenting users with many different skin-tones.

Yogarajah et al. (2010) proposed a skin segmentation algorithm, which calculates dynamic threshold values considering the color information of the detected face regions. In this approach fixed decision boundaries (or fixed threshold) classification approaches are successfully applied to segment human skin. These fixed thresholds mostly failed in two situations as they only search for a certain skin color range. (i) Any non-skin object may be classified as skin if non-skin object's color values belong to fixed threshold range. (ii) Any true skin may be mistakenly classified as non-skin if that skin color values do not belong to fixed threshold range. However, this method fails on varying illumination condition.

Yin et al. (2011) tried to detect adult images by considering color, texture and geometrical features of an image. The idea that was implemented in that research paper was color filtering the image to determine candidate skin regions, then the coarse degree of pixels of candidate skin regions was calculated for each pixel and lastly, fractal dimension of all the rest big enough skin regions was calculated and after a couple of iterations, a threshold was picked to use in decision making. 


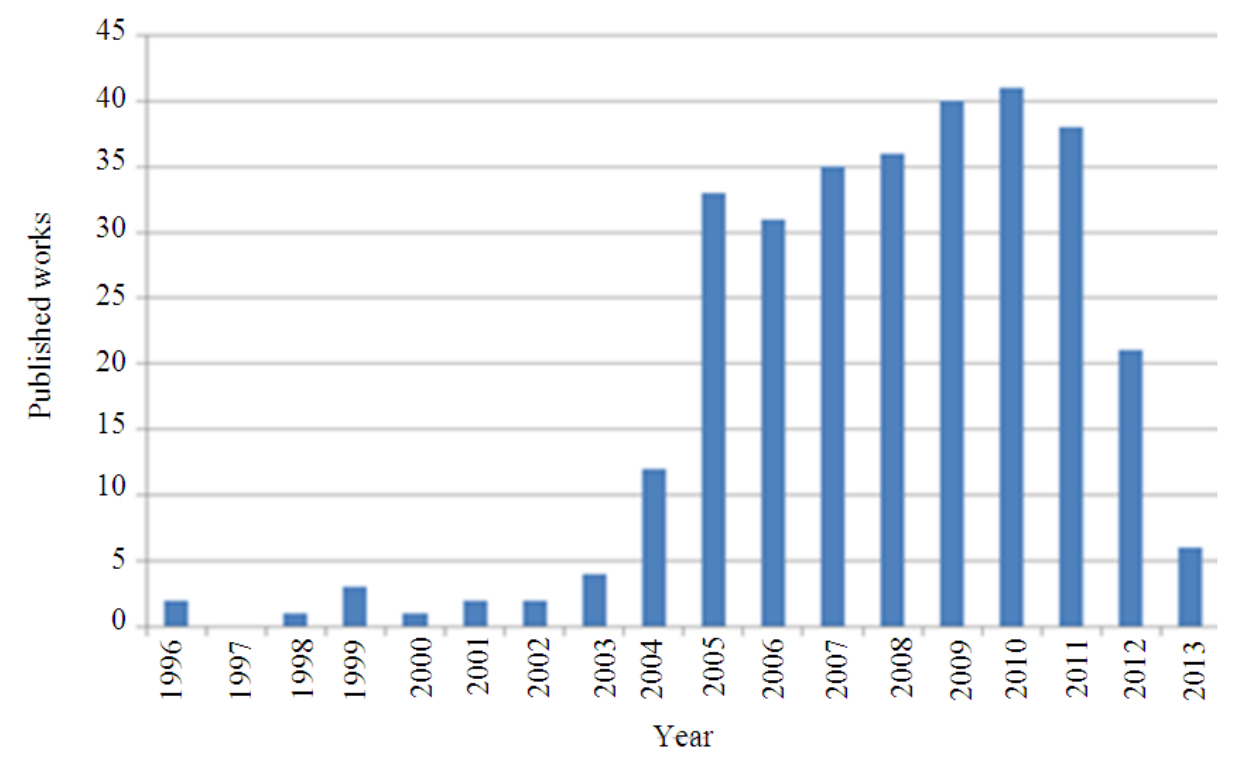

Fig. 1. Published works (1996-2013) on content based pornography detection

Jedynak et al. (2003) proposed a statistical model for skin detection. The maximum entropy model is used to infer the skin models from the data set. It has been shown that maximum entropy modeling can lead to an efficient algorithm for a supervised image segmentation problem. The Bethe Tree approximation and Belief Propagation algorithm are utilized in their work to approximate the probability for skin at pixel locations.

Zeng et al. (2004) proposed an intelligent adult image detector, called Image Guarder, which can automatically analyze the image content, efficiently recognize adult images. In order to speed up recognition, a two layers recognition framework is adopted. An adaptive statistical color model is developed to detect the skin pixel in variant illumination environment. Smooth character is used to test the skin texture feature after skin color detection.

Abadpour and Ksaei (2005) articulated a work on pixel-based skin detection for pornography filtering. In this work they investigated 21 color spaces in all of their possible representations for pixel-based skin detection in pornographic images. In connection of this work later Hedieh et al. (2007) proposed a similar work on a Boosted Skin Detection Method based on Pixel and Block information. In this work the authors implemented boosted pixel-based skin detector architecture.

It is to be noted that most of the works devoted to skin detection and color space model but there are lots of shortcomings. Presence of skin like objects and highly exposed skins without objectionable part may increase false positive or negative ratio. Hence, now a day researchers are concentrating on both skin and erotogenic body parts.

\subsection{Region Based Skin Detection}

Zheng et al. (2004) investigated adult image detection based on the shape features of skin regions using multi-Bayes classifier. The approach was to identify human shape feature and fed to a boosted classifier (C4.5) to decide whether or not the skin regions represent a nude. It has been demonstrated that using combination of different shape descriptors can enhance the performance of shape classification.

\section{EROTOGENIC BODY PARTS DETECTION}

\subsection{Nipple Detection}

Fuangkhon and Tanprasert (2005) presented an object detection using image processing and neural network entitled "nipple detection for obscene pictures". The authors claimed that the detection rate was $65.4 \%$. So far this paper was the only paper on nipple detection. In Wang et al. (2010) proposed another robust method entitled "Automatic Nipple Detection Using Shape and Statistical Skin Color Information". In this study a new approach on nipple detection for adult content recognition presented and it combines the advantage of 
Adaboost algorithm that is rapid speed in object detection and the robustness of nipple features for adaptive nipple detection. The detection rate of this approach was $75.6 \%$.

\subsection{Naked Body Detection}

Wang et al. (2009) depicted a new method for identifying adult images based on naked body detection. In this work they considered navel and body features, which are composed by trunk, limb and face. The idea behind in this method was to consider the body as a combination of predefined key rectangles. The intersection of the key rectangles is the navel. Firstly, the algorithm recognizes the location of navel in an image. Then it constructs the feature vector of key rectangles. Finally, it classifies the features by Forward Propagation neural network to determine whether there is a naked body in the image or not. Experiments show that the algorithm can recognize nude images accurately and the recognizing speed can satisfy the requirement of practical systems.

\subsection{Key Parts Detection}

Pengyu and Jie (2013) presented a novel pornographic filtering method based on human key parts detection. It is published in 2013. In this study the authors described extraction of Haar-like features which depicts local grayscale distribution and then train the classifier with AdaBoost learning algorithm. Histogram of Oriented Gradient (HOG) and Gray level Cooccurrence Matrix (GLCM) features extracted from human key parts for performance.

\subsection{Face and Trunk Detection}

Shen et al. (2007) illustrated a method based on face and trunk detection. This paper compared with several deficiencies of the previous approaches, which detected by the features of skin-color only, the paper presents a novel algorithm based on the face and trunk detection and through the recognizing the erotogenic part in the image, they implemented the algorithm and construct a simple and fast pornographic image classifier system based on a decision tree. The detection rate is $80.31 \%$.

\section{COMPARATIVE EVALUATION}

In this section some well-known and widely used image screening techniques will be evaluated following its limitations and possible extensions. It is to be noted that pornography detection has been carried out using human skin likelihood ratio and erotogenic body parts (navel, nipple, pubic hair, breast, trunk, limb) detection. It has been observed that the detection rate would be higher by using hybrid model of skin ratios and erotogenic body parts. In addition usages of suitable classifiers (SVM and Adaboost) are also responsible for good performance. Different skin types such as white, pink, yellow, brown and dark can be classified by a good classifier in accordance with variety of illumination conditions on skins. The evaluation carried out in terms of True Positive Rate (TPR) and False Positive Rate (FPR). It is to be noted that all experiments carried out using different datasets. So, it is quiet impossible to obtain a proper evaluation of all methods since they are not evaluated on the same train and test datasets.

Every work tried to address certain problems to detect pornography, for example varying illumination condition Yin et al. (2011), increase detection rate using the same tools and datasets but different features, problems of close-up images and varying illumination conditions Yogarajah et al. (2010). If we consider erotogenic human body parts and Adaboost as a classifier then the work Pornographic Image Filtering Method Based on Human Key Parts (Haar like features) Pengyu and Jie (2013) showed the best performance (94.13\% detection rate).

Works in section 3 and 4 organized according to the following two principle detection techniques: (i) Skin segmentations and (ii) erotogenic body parts. Both features utilized some color space model, image segmentation and machine learning tools. Selection of appropriate color space is very important for human skin detection. As discussed in section $3 \mathrm{YCbCr}$ color model is the best choice of skin detection for all types of ethnicity. For image segmentation, features are another important tool. Most scholars used skin color model as feature descriptor. Some well known features like HOG (Histogram of Oriented Gradient) for upright person detection, GLCM (Gray Level Cooccurrence Matrix and Gabor wavelet for texture based skin detection can also be applicable for better detection of pornography. All feature descriptor working in certain constraint. It is impossible to find a unique feature for all types of objectionable images.

\section{OPEN RESEARCH ISSUES}

In this section some emerging open research issues will be illustrated. 


\subsection{Choice of Classifiers and Feature Selection}

The following would be considered as open research issue. Integrate a fast face detector and add some extra features such as face-skin divided by body-skin and biggest skin patch connected. Make use of skin adaptive models. Find a compact and more knowledgeable way to characterize the color information like incorporating color variance. Hybrid classifiers (SVM and Adaboost) can be used for better performance. Sometimes censored images may also be useful for education purposes or may be categorized according to age and culture. These things will be done perfectly by incorporation of knowledge base. Necessary steps could be done, training the stored and auto updateable database. In addition unsupervised transfer learning Pan and Yang (2010) can be applicable instead of traditional supervised classification because it is impossible to classify specific nude picture using a predefined set of dataset. Since there are more and more different kinds of objectionable pictures available, the traditional machine learning algorithm maybe inefficient to find new type of nude picture based on the old training datasets. In this situation transfer learning can be applicable to assist the discovery procedure.

\subsection{Multimedia Cloud Computing for Obscenity Detection}

Multimedia cloud computing is now a popular research topic due to wide spread information sharing and up gradation of network bandwidth. There are many works has been accomplished in cloud computing handling multimedia (Audio, video, image) but very little works in processing content based multimedia (image and video). Almeer (2012); Ramos-Pollan et al. (2012); Pereira et al. (2010); Zhu et al. (2004) and Chris et al. (2011). Still now there are only three methods introduced to process contents of images in cloud computing. (i) Hadoop Image Processing Interface (HIPI) Chris et al. (2011) (ii) Hadoop SEQENCE files Hadoop, 2013 and (iii) BIGS Ramos-Pollan et al. (2012). Sequence files perform better than standard applications for small files, but must be read serially and take a very long time to generate Chris et al. (2011). HIPI Image Bundle have similar speeds to Sequence files, do not have to be read serially and can be generated with a MapReduce program Chris et al. (2011). Additionally, HIPI Image Bundles are more customizable and are mutable, unlike Sequence files. For instance, HIPI has the ability to only read the header of an image file using HIPI Image Bundles, which would be considerably more difficult with other file types. Those three methods of handling images in Hadoop can process images based on some ground truth and image statistics. No work efficiently process images according to appropriate machine learning scenario. In SVM, 2013 the use of parallel SVM in Hadoop has been elucidated but still there in need rigorous analysis. In this perspective, scholars can think about how to fit machine learning strategies in Cloud Computing architecture.

\section{CONCLUSION}

Most of the existing works are based on skin color region detection which can't perfectly recognize whether an image contain obscenity in general sense. The reason for this is that, there are many skins like pixels such as beach photos, human skin like animal's fur, skin colored painting which enable false positive rate. In addition all existing works perform well on particular set of image dataset. It has been observed from table that, in spite of successfully applying skin color segmentation and geometrical structure of human body, eventually it was failed to detect naked body perfectly due to absence of machine learning tools Fleek et al. (1996). On the other hand, using skin color segmentation and machine learning tools improve detection rate significantly. Here choice of appropriate color models, skin detection algorithms and classifiers are the factors of performance. The performance of pornography detection has been dramatically changed using human body parts specifically erotogenic parts. In this case choice of proper color model, skin segmentation algorithms, classifiers and human erotogenic body parts are the factors.

In all cases a specific dataset has been applied for test images and hence the detection could not satisfy for all types of arbitrary nude images. Because there are huge amount of nude images available in their different pose, angle, illumination condition, partial occlusion, highly and partially exposed form. In this case unsupervised transfer learning could be a solution because it creates new data sets from already learned old datasets and thus would perform well on random unlabelled nude picture identification.

There are some common trends in every detection algorithms taking consideration of accuracy and performance. These two things are inversely proportional. To minimize this challenges parallel and distributed systems can be applicable. If we consider accuracy of obscenity detection then should pay attention for substantial improvement of existing image based 
obscenity detection or if we consider a suitable existing method and want to improve the performance of the system then we should pay attention for applying parallel and distributed system. A cloud computing system can be utilized in this perspective. Content-based image processing in the cloud is still an open research issue. The reason for this is that in cloud computing paradigm only text-based data can be recognizable and there is no such built in tool to handle byte oriented image data Almeer (2012). Some scholars indicated to handle this problem using Hadoop SEQUENCE file Hadoop, 2013. But this technique also not had been proved yet. Recently there are two tools has been deployed to process large-scale image such as HIPI Chris et al. (2011) and BIGS Ramos-Pollan et al. (2012). These two techniques devoted to process some special images for example remote sensing and medical images. It is not sure whether it can work on objectionable images or not. There is another problem of processing images in distributed environment. Image can't be split during the processing phase because it affects the quality of the original image during merging Almeer (2012). If those issues could be resolved then performance of CBPD (Content based pornography detection) will increase significantly.

\section{ACKNOWLEDGMENT}

This research was supported in part by Shenzhen Technical Project (grant no. HLE201104220082A) and National Natural Science Foundation of China (grant no. 61105133) and Shenzhen Public Technical Platform (grant no. CXC201005260003A).

\section{REFERENCES}

Abadpour, A. and S. Kasaei, 2005. Pixel-based skin detection for pornography filtering. Iranian J. Electr. Electron. Eng., 1: 21-41.

Almeer, M.H., 2012. Cloud hadoop map reduce for remote sensing image analysis. J. Emerg. Trends Comput. Inform. Sci., 3: 637-644.

Alshamasin, M., R. Al-kasasbeh, A. Khraiwish, Y. Alshiboul and D.E. Skopin, 2009. Acceleration of image processing using new color model. Am. J. Applied Sci., 6: 1015-1020. DOI: 10.3844/ajassp.2009.1015.1020

Brown, D., I. Craw and J. Lewthwaite, 2001. A SOM based approach to skin detection with application in real time systems. University of Aberdeen.
Caetano, T.S. and D.A.C. Barone, 2001. A probabilistic model for the human skin color. Proceedings of the 11th International Conference on Image Analysis and Processing, Sept. 16-18, IEEE Xplore Press, Palermo, p: 279-283. DOI: 10.1109/ICIAP.2001.957022

Chris, S., L. Liu, A. Sean and L. Jason, 2011. HIPI: A hadoop image processing interface for image-based mapreduce tasks. University of Virginia.

Fleek, M.M., D.A. Forsyth and C. Bregler, 1996. Finding naked people. Proceedings of the 4th European Conference on Computer Vision, Apr. 15-18, Springer Berlin Heidelberg, Cambridge, UK., pp: 593-602. DOI: 10.1007/3-540-61123-1_173

Fuangkhon, P. and T. Tanprasert, 2005. Nipple detection for obscene pictures. Proceedings of the 5th WSEAS International Conference on Signal, Speech and Image Processing, (SIP' 05), World Scientific and Engineering Academy and Society (WSEAS) Stevens Point, Wisconsin, USA., pp: 242-247.

Geetha, P. and V. Narayanan, 2008. A Survey of Content-Based Video Retrieval. J. Comput. Sci., 4: 474-486. DOI: 10.3844/jcssp.2008.474.486

Hedieh, S., M. Najafi and S. Kasaei, 2007. A boosted skin detection method based on pixel and block information. Proceedings of the 5th International Symposium on Image and Signal Processing and Analysis, Sept. 27-29, IEEE Xplore Press, Istanbul, pp: 146-151. DOI: 10.1109/ISPA.2007.4383680

Jaganathan, Y. and I. Vennila, 2013. Feature dimension reduction for efficient medical image retrieval system using unified framework. J. Comput. Sci., 9: 1472-1486. DOI: 10.3844/jcssp.2013.1472.1486

Jedynak, B., H Zheng and M. Daoudi, 2003. Statistical models for skin detection. Proceedings of the Conference on Computer Vision and Pattern Recognition Workshop, Jun. 16-22, IEEE Xplore Press, Madison, Wisconsin, USA., pp: 92-92. DOI: 10.1109/CVPRW.2003.10094

Kakumanu, P., S. Makrogiannis and N. Bourbakis, 2007. A survey of skin-color modeling and detection methods. Patt. Recogn., 40: 1106-112. DOI: 10.1016/j.patcog.2006.06.010

Kjeldsen, R. and J. Kender, 1996. Finding skin in color images. Proceedings of the 2nd International Conference on Automatic Face and Gesture Recognition, Oct. 14-16, IEEE Xplore Press, Killington, VT., pp: 312-317. DOI: 10.1109/AFGR.1996.557283 
Pan, S.J. and Q. Yang, 2010. A survey on transfer learning. IEEE Trans. Knowl. Data Eng., 22: 13451359. DOI: 10.1109/TKDE.2009.191

Pengyu, N. and H. Jie, 2013. Pornographic image filtering method based on human key parts. Proceedings of the International Conference on Information Technology and Software Engineering, (TSE' 13), Springer Berlin Heidelberg, pp: 677-688. DOI: 10.1007/978-3-642-34531-9_71

Pereira, R., M. Azambuja, K. Breitman and M. Endler, 2010. An architecture for distributed high performance video processing in the cloud. Proceedings of the IEEE 3rd International Conference on Cloud Computing, Jul. 05-10, IEEE Xplore Press, Miami, FL., pp: 482-489. DOI: 10.1109/CLOUD.2010.73

Ramos-Pollan, R., F.A. Gonzalez, J.C. Caicedo, A. CruzRoa and J.E. Camargo et al., 2012. BIGS: A framework for large-scale image processing and analysis over distributed and heterogeneous computing resources. Proceedings of the IEEE 8th International Conference on E-Science, Oct. 8-12, IEEE Xplore Press, Chicago, IL., pp: 1-8. DOI: 10.1109/eScience.2012.6404424

Rossotti, H., 1983. Colour: Why the World isn't Grey. 1st Edn., Princeton University Press, Princeton, ISBN-10: 0691023867, pp: 239.

Shen, X., W. Wei and Q. Qian, 2007. The filtering of internet images based on detecting erotogenic-part. Proceedings of the 3rd International Conference on Natural Computation, Aug. 24-27, IEEE Xplore Press, Haikou, pp: 732-736. DOI: 10.1109/ICNC.2007.725

Taqa, A.Y. and H.A. Jalab, 2010. Increasing the reliability of fuzzy inference system-based skin detector. Am. J. Applied Sci., 7: 1129-1138. DOI: 10.3844/ajassp.2010.1129.1138

Vezhnevets, V., V. Sazonov and A. Andreeva, 2003. A survey on pixel-based skin color detection techniques. Moscow State University.

Wang, X., C. Hu and S. Yao, 2009. An adult image recognizing algorithm based on naked body detection. Proceedings of the ISECS International Colloquium on Computing, Communication, Control and Management, Aug. 8-9, IEEE Xplore Press, Sanya, pp: 197-200. DOI: 10.1109/CCCM.2009.5267781
Wang, Y., J. Li, H.L. Wang and Z.J. Hou, 2010. Automatic nipple detection using shape and statistical skin color information. Proceedings of the 16th International Conference on Advances in Multimedia Modeling, Jan. 6-8, Springer-Verlag Berlin, Chongqing, China, pp: 644-649. DOI: 10.1007/978-3-642-11301-7_63

Wong, K.W., K.M. Lam and W.C. Siu, 2003. A robust scheme for live detection of human faces in color images. Signal Process. Image Commun., 18: 103114. DOI: 10.1016/S0923-5965(02)00088-7

Yin, H., X. Xu and L. Ye, 2011. Big skin regions detection for adult image identification. Proceedings of the Workshop on Digital Media and Digital Content Management, May 15-16, IEEE Xplore Press, Hangzhou, pp: 242-247. DOI: 10.1109/DMDCM.2011.36

Yogarajah, P., J. Condell, K. Curran, A. Cheddad and P. McKevitt, 2010. A dynamic threshold approach for skin segmentation in color images. Proceedings of the IEEE International Conference on Image Processing, Sep. 26-29, IEEE Xplore Press, Hong Kong, pp: 2225-2228. DOI: 10.1109/ICIP.2010.5652798

Zeng, W., W. Gao, T. Zhang and Y. Liu, 2004. Image guarder: An in telligent detector for adult images. Institute of Computing Technology.

Zheng, Q.F., M.J. Zhang, G. Wen and W.Q. Wang, 2004. Shape-based adult images detection. Proceedings of the IEEE 1st Symposium on MultiAgent Security and Survivability, Dec. 18-20, IEEE Xplore Press, pp: 150-153. DOI: 10.1109/ICIG.2004.128

Zhu, Q., K.T. Cheng, C.T. Wu and Y.L. Wu, 2004, Adaptive learning of an accurate skin-color model. Proceedings of the 6th IEEE International Conference on Automatic Face and Gesture Recognition, May 17-19, IEEE Xplore Press, pp: 37-42. DOI: 10.1109/AFGR.2004.1301506 